DOI: $10.21767 / 2576-3903.100006$

\section{C-Licorice in Human Cancer: Still Many Times to Use it?}

Received: November 11, 2016; Accepted: November 18, 2016; Published: November 21, 2016

Licorice or liquorice (Glycyrrhiza glabra L.) is a perennial plant used in food and as medicine for thousands of years. It belongs to the genus Glycyrrhiza, which comprises 2 other original plants with similar properties, Glycyrrhiza uralensis Fisch. and Glycyrrhiza inflate Bat. Licorice derives from the ancient Greek term "glukurrhiza", composed by 2 terms: glukus meaning "sweet" and rhiza meaning "root" [1]. It is native of Southern Europe and Asia, where given its sweet favor, licorice is commonly used in many foods, condiments and confectionery. Moreover, licorice has medicinal properties: traditional and ethno-botanical uses for human diseases, such as those affecting respiratory, gastrointestinal, cardiovascular, genital-urinary, epidermal systems, are well documented, giving to licorice a valuable weight in medicine [2-4]. In more recent years, licorice has been widely and scientifically studied as a therapeutic agent in preclinical models; although not all of it uses can be supported by scientific evidence [5-7]. In addition to it is noteworthy that licorice at high doses can have adverse effects, due to its aldosterone-like effect which suppresses the renin-angiotensin-aldosterone axis and causes hypertension, hypokalaemia, metabolic alkalosis and volume expansion $[8,9]$.

To date, more than 20 terpenoids and 300 flavonoids have been isolated from licorice. Many were investigated and only those possessing pharmacological activities were extensively studied [10]. The most analyzed bioactive compounds are probably glycyrrhetinic acid, glycyrrhizin (also called glycyrrhizic acid) and 11-deoxyglycyrrhetinic acid, although many other were investigated (for example liquiritin, isoliquiritin, liquiritigenin, isoliquiritigenin, glabridin, dehydroglyasperin C, dehydroglyasperin $D$, carbenoxolone, etc.). Glycyrrhizin is a triterpenoid 50 times more sweetener than sucrose and shows a slower onset than sugar but can persist in the mouth for a longer time [11]. Glycyrrhizin can act on many key cell factors resulting in anti-inflammatory response. Its effects can induce modulation of PI3K signalling pathway, antioxidant system (with ROS reduction), cytokines and immune system $[10,12]$. In addition, glycyrrhizin and glycyrrhetinic acid possess many other positive effects on human health, such as antitumor, antimicrobial, antiviral, antidiabetic, immunoregulatory, hepatoprotective activities, contributing also to protection and recovery of the endocrine, respiratory, gastrointestinal, nervous and cardiovascular system [13-15]. Among potential benefits, antitumor activity of licorice

\author{
Raffaele Pezzani ${ }^{1,2}$ \\ 1 OU Endocrinology, DIMED, University of \\ Padova, via Ospedale, Padova, Italy \\ 2 AIROB, Associazione Italiana per la \\ Ricerca Oncologica di Base, Padova, Italy
}

\author{
Corresponding author: \\ Dr. Rafele Pezzani
}

$\equiv$ raffaele.pezzani@unipd.it

\begin{abstract}
OU Endocrinology, DIMED, University of Padova, via Ospedale 105, 35128

Padova, Italy.
\end{abstract}

Tel: 390498213018

Citation: Pezzani R. C-Licorice in Human Cancer: Still Many Times to Use it?. J Neoplasm 2016, 1:2

falls in the concept of using natural products to fight against cancer. We know that cancer is the second leading cause of death in Western Countries and still represents a great challenge for scientific research [16]. In this regard, every effort to combat cancer should be improved: herbal medicines mean another useful tool to help antitumor investigation. Licorice root extracts or biosynthetic compounds derived from licorice have been extensively explored in preclinical or basic research, underlining very interesting and potentially decisive effects in cell and animal models [7]. However, as for many other bioactive compounds (natural or artificial), the use of licorice has been never exhaustively investigated in clinical trials involving human cancer. Indeed, licorice entered in no more than 30 human studies and only 7 clinical trials attempted to investigate licorice in cancer [17]. They are divided into 2 groups: the first (only one study) analysed the use of licorice root extract associated with docetaxel in treating patients with metastatic prostate cancer, not responding to hormone therapy. Unfortunately, the study was closed early due to slow accrual and insufficient data could be collected to assess the original primary outcomes: a) percentage of patients with PSA response such as decline from baseline value by $>50 \%$, or normalization of PSA (defined as PSA less than $0.2 \mathrm{ng} / \mathrm{ml}$ ), b) efficacy and toxicity of licorice root in combination with docetaxel in patients with hormone-refractory prostate cancer. The second group used PHY906 in colorectal, pancreatic, hepatocellular cancer. PHY906 is a powder obtained from different plants sold 
as a health food supplement in the United States, used in China, Taiwan and other Asian countries as traditional Chinese medicine for hundreds of years. PHY906 contains 4 traditional Chinese herbal medicines, Glycyrrhiza uralensis Fisch, Paeonia lactiflora Pall, Scutellaria baicalensis Georgi, and Ziziphus jujuba Mill and is currently being developed as an adjuvant for chemotherapy [18]. This second group used a combination of plants, rather than pure licorice extract, consequently the quantity of Glycyrrhiza extract is very low and the potential effects could not be imputable to Glycyrrhizaalone. However, taking this kind of extract (PHY906) into account, 3 studies are active (recruiting), 2 are completed and 1 is terminated. Only one study reported results: a phase I/ II, multi-center, open-label, dose-escalation, safety and efficacy study of PHY906 plus capecitabine in patients with advanced pancreatic carcinoma. The results indicated that the primary outcome (median progression free survival) was 10.1 weeks (with a full range from 0.4 to 54.1 weeks) and the secondary outcome (median overall survival) was 21.6 weeks (with a full range from 0.4 to 84.1 weeks).

As it is perceivable, no substantial or concrete data can emerge from the review of clinical trials investigating the use of licorice in human cancer. This issue suggests that more work is still necessary to explore the potential effect of licorice in cancer: we are at the very beginning of the story. Moreover, it seems that there are many difficulties or problems that researchers face with probably of the primary one still remain funds. Indeed, licorice is inexpensive and no patentee seems to be suitable for it. Which pharma company would invest on it? Our hope is that public research could fill in this gap and that scientific world keeps in mind the ultimate aim of research, i.e., to provide new treatment options to cure human diseases. 


\section{References}

1 https://en.oxforddictionaries.com/definition

2 Chopra RN, Chopra IC, Handa KL, Kapur LD (1958) Indigenous drugs of India. Calcutta, India.

3 Fiore C, Eisenhut M, Ragazzi E, Zanchin G, Armanini D (2005) A history of the therapeutic use of liquorice in Europe. Journal of Ethnopharmacology 99: 317-324.

4 Thompson C (1930) Assyrian prescriptions for treating bruises or swelling.

5 Shibata SA (2000) Drug over the millennia: Pharmacognosy, chemistry, and pharmacology of licorice. Yakugaku zasshi: Journal of the Pharmaceutical Society of Japan 120: 849.

6 Armanini D, Fiore C, Mattarello MJ, Bielenberg J, Palermo M (2002) History of the endocrine effects of licorice. Experimental and clinical endocrinology \& diabetes: official journal, German Society of Endocrinology and German Diabetes Association 110: 257-261.

7 Roohbakhsh A, Iranshahy M, Iranshahi M (2016) Glycyrrhetinic acid and its derivatives: Anti-cancer and cancer chemopreventive properties: Mechanisms of action and structure-cytotoxic activity relationship. Current Medicinal Chemistry 23: 498-517.

8 Armanini D, Lewicka S, Pratesi C, Scali M, Zennaro MC, et al. (1996) Further studies on the mechanism of the mineralocorticoid action of licorice in humans. Journal of Endocrinological Investigation 19: 624-629.

9 Di Lorenzo C, Ceschi A, Kupferschmidt H, Lude S, De Souza Nascimento E, et al. (2015) Adverse effects of plant food supplements and botanical preparations: a systematic review with critical evaluation of causality. British Journal of Clinical Pharmacology 79: 578-592.

10 Yang R, Wang LQ, Yuan BC, Liu Y (2015) The pharmacological activities of licorice. Planta Medica 81: 1654-1669.

11 Ahamed A, Tsurumi S, Ozaki M, Amakawa T (2001) An artificial sweetener stimulates the sweet taste in insect: dual effects of glycyrrhizin in Phormia regina. Chemical senses 26: 507.

12 Isbrucker RA, Burdock GA (2006) Risk and safety assessment on the consumption of Licorice root (Glycyrrhiza sp.), its extract and powder as a food ingredient, with emphasis on the pharmacology and toxicology of glycyrrhizin. Regulatory Toxicology and Pharmacology 46: 167-192.

13 Feng X, Ding L, Qiu F (2015) Potential drug interactions associated with glycyrrhizin and glycyrrhetinic acid. Drug Metabolism Reviews 47: 229-238.

14 Obolentseva GV, Litvinenko VI, Ammosov AS, Popova TP, Sampiev AM (1999) Pharmacological and therapeutic properties of licorice preparations (A review). Pharmaceutical Chemistry Journal 33: 427-434.

15 Kao TC, Wu CH, Yen GC (2014) Bioactivity and potential health benefits of licorice. Journal of Agricultural and Food Chemistry 62: 542-553.

16 www.cancer.org

17 www.clinicaltrials.gov

18 Qi F, Zhao L, Zhou A, Zhang B, Li A, et al. (2015) The advantages of using traditional Chinese medicine as an adjunctive therapy in the whole course of cancer treatment instead of only terminal stage of cancer. Bioscience Trends 9: 16-34. 